\title{
Imeglimin: A New Promising and Effective Weapon in the Treatment of Type 2 Diabetes
}

\author{
John Doupis, Neoklis Baris and Konstantinos Avramidis \\ Department of Internal Medicine and Diabetes, Salamis Naval and Veterans Hospital, Salamis Naval Base, Attiki, Greece
}

DOI: https://doi.org/10.17925/EE.2021.17.2.88

meglimin is a novel molecule currently under development for the treatment of type 2 diabetes mellitus, and is the first agent of the 'glimin' class of glucose-lowering medication. It has a unique mechanism of action that targets the three main pathophysiologic components

of type 2 diabetes: impaired glucose uptake by muscle tissue, excess hepatic gluconeogenesis and increased $\beta$-cell apoptosis. To date, imeglimin has been evaluated in many preclinical and clinical trials and has shown to have notable antihyperglycaemic effects, such as statistically significant reductions in glycated haemoglobin, fasting plasma glucose and other glycaemic parameters. The encouraging tolerability profile, combined with its efficacy, could make it suitable as a monotherapy or in combination with other classes of antidiabetic agents, hopefully in the near future.

\section{Keywords}

Type 2 diabetes mellitus, imeglimin, mechanism of action, clinical trials

Disclosures: John Doupis, Neoklis Baris and Konstantinos Avramidis have no financial or non-financial relationships or activities to declare in relation to this article.

Review process: Double-blind peer review.

Compliance with ethics: This study involves a review of the literature and did not involve any studies with human or animal subjects performed by any of the authors.

Data availability: Data sharing is not applicable to this article as no datasets were generated or analysed during the writing of this article.

Authorship: The named authors meet the Internationa Committee of Medical Journal Editors (ICMJE) criteria for authorship of this manuscript, take responsibility for the integrity of the work as a whole, and have given final approval for the version to be published. Access: This article is freely accessible at touchENDOCRINOLOGY.com @ T Touch Medical Media 2021. Received: 1 February 2021

Accepted: 21 April 2021

Published online: 10 November 2021

Citation: touchREVIEWS in

Endocrinology. 2021;17(2):88-91

Corresponding author: John Doupis, Department of Internal Medicine and Diabetes, Salamis Naval and Veterans Hospital, Salamis Naval Base, 18900, Salamis, Attiki, Greece. E: john.doupis@harvard-alumni.org

Support: No funding was received in

the publication of this article.
Imeglimin is a novel molecule currently under development for the treatment of type 2 diabetes mellitus, and is the first agent of the 'glimin' class of glucose-lowering medication. Optimal glucose control in type 2 diabetes is a prerequisite for correcting defects in both insulin secretion and insulin sensitivity. ${ }^{1}$ Imeglimin presents a unique mechanism of action that targets the three main pathophysiologic components of type 2 diabetes: impaired glucose uptake by muscle tissue, excess hepatic gluconeogenesis and increased $\beta$-cell apoptosis. Recent phase II and phase III trials demonstrated the efficacy of imeglimin, either as a monotherapy or in combination with other glucose-lowering drugs, in improving glycated haemoglobin ( $\mathrm{HbA} 1 \mathrm{C})$ and fasting plasma glucose (FPG) levels. ${ }^{2}$ This is a narrative review that subjectively summarizes the available data found on PubMed, illustrating the potential for imeglimin to be a promising candidate for the treatment of type 2 diabetes in all stages of the disease spectrum. For the purposes of the concise nature of this review, we chose to focus on imeglimin's impact on HbA1c and FPG.

\section{Pharmacokinetics: Chemical properties}

Imeglimin is a tetrahydrotriazine compound. Its chemical name is (6R)-(+)-4-dimethylamino-2imino-6-methyl-1,2,5,6-tetrahydro-1,3,5-triazine hydrochloride, and its primary site of action is the mitochondria of the aerobic cells, where it alters the oxidative phosphorylation, serving also as a substrate for organic cation transporters. ${ }^{3}$ There are signs to suggest that imeglimin induces an insulin-dependent protein kinase B phosphorylation increase, in both liver and muscle cells. In the liver, imeglimin changes the flow of substrates and rebalances the pathways, favouring complex II, inhibiting complex I (competitive mitochondrial inhibition) and restoring complex III functions. As a result, it reduces liver steatosis and reactive oxygen species (ROS) produced in mitochondria, and increases hepatic 3-hydroxyacetyl-COA dehydrogenase activity. ${ }^{4}$

Imeglimin demonstrates a moderate intestinal absorption (50-80\%) through the mechanism of active paracellular absorption; however, increased dosages of oral imeglimin have resulted in decreased intestinal absorption, likely due to the saturation of the active transport mechanism. ${ }^{5}$ Consequently, the distribution of the drug through the bloodstream is fast, and plasma protein binding is low. Furthermore, there is no evidence of cytochrome P450 inhibition or induction. ${ }^{5}$ Imeglimin is excreted through the kidneys and mostly remains unchanged. ${ }^{6} \mathrm{~A}$ recent study by Chevalier et al. has indicated that it is safe and well tolerated in patients with moderate hepatic impairment, as the increase in maximum plasma concentration and the area under the curve (AUC) of concentration/time was not reported as clinically significant. ${ }^{6}$

\section{Mechanism of action and preclinical studies}

Imeglimin seems to tackle three main pathways of type 2 diabetes by either reversing or ameliorating them. These include i) excessive hepatic glucose production; ii) impaired peripheral glucose uptake by insulin-sensitive tissues; and iii) pancreatic $\beta$-cell dysfunction. It is a unique drug because of its multiple actions, which seem to make imeglimin a promising new antidiabetic agent. Imeglimin's main proposed mechanisms of action are summarized below. 


\section{Inhibition of hepatic glucose production}

Imeglimin has shown an inhibitory effect in glucose production in both isolated rat liver cells and rat liver slices. ${ }^{4,5}$ Moreover, imeglimin elicited a reduction in glucose produced by the isolated cells in a concentration-dependent pattern, with reductions ranging from $9 \%$ (for $0.25 \mathrm{mmol} / \mathrm{L}$ ) to $80 \%$ (for $1.5 \mathrm{mmol} / \mathrm{L}$ ), which is comparable to the results of metformin in its highest dosages.? In liver slices, the inhibition of glucose production was also apparent; in a dose-dependent pattern, the reduction ranged from $14 \%$ (for $2.5 \mathrm{mmol} / \mathrm{L}$ ) to $84 \%$ (for $10 \mathrm{mmol} / \mathrm{L}){ }^{5}$ On a molecular level, imeglimin achieved these results by downregulating phosphoenolpyruvate carboxykinase (PEPCK) and glucose-6-phosphatase (G6Pase) in isolated hepatocytes from rats, and by inhibiting lactic acidosis via the mitochondrial-dependent pathway. ${ }^{3,4}$

\section{Stimulation of skeletal muscle glucose uptake}

In muscle cell cultures, imeglimin has shown effectiveness by inducing glucose uptake by the muscle cells, which was statistically significant at a dose of $0.5 \mathrm{mmol} / \mathrm{L}(\mathrm{p}<0.01)$, with up to a 3.3-fold increase at the maximum dose given ( $2 \mathrm{mmol} / \mathrm{L})$, compared with control.? In vivo, in soleus and gastrocnemius muscle from streptozotocin diabetic rats, imeglimin showed a statistically significant $(p<0.01)$ increase in glucose uptake even at the lowest dose $(25 \mathrm{mg} / \mathrm{kg})$, restoring the uptake to normal for diabetic rats at $50 \mathrm{mg} / \mathrm{kg}$ and $100 \mathrm{mg} / \mathrm{kg}$ ( $p<0.05$ and $p<0.01$, respectively). There are various proposed molecular pathways for the action of imeglimin on insulin sensitivity, even though they are not fully understood. One proposed way is the increase in Akt (protein kinase B) phosphorylation, which passes the insulin signal transduction; another possible pathway might include glucose transporter-4 expression and the regulation of insulin receptor substrate phosphorylation., ${ }^{3,4}$

\section{Reversal of pancreatic $\beta$-cell dysfunction}

There are several studies that demonstrate improved glucose-stimulated insulin secretion in animal models. In the perfused pancreas of streptozotocin diabetic rats, imeglimin provoked insulin secretion, solely in the presence of glucose. This effect did not appear in the absence of glucose, showing a strong glucose-stimulated pattern of action. In a study in non-diabetic Wistar rats, insulin production increased by $25 \%$ in the imeglimin group compared to the control group, at the highest levels of hyperglycaemia during a hyperglycaemic clamp. On the other hand, sitagliptin and repaglinide induced an increase of $25 \%$ and $11 \%$, respectively. This effect was absent at basal glycaemic levels and the first stage of hyperglycaemia. In diabetic neonatal streptozotocin rats, imeglimin provoked an increase in insulin levels at all glucose levels tested, with an increase of $48 \%$ at basal levels, $62 \%$ at the first stage of hyperglycaemia and $68 \%$ at the second stage.? Based on a recent study on awake rodents, 1 week of treatment with imeglimin showed an increase in glucose-dependent insulin secretion, with an estimated $36 \%$ increase in $\beta$-cell glucose sensitivity. It is also noteworthy that glucagon levels were not affected. ${ }^{8}$ On a molecular basis, postprandial insulin secretion is mediated by a nicotinamide adenine dinucleotide-dependent mechanism. $3 ., 4$

Imeglimin has also shown promising results as potentially protective from pancreatic $\beta$-cell apoptosis and glucose-induced $\beta$-cell death, suggesting that it may help preserve the functional $\beta$-cell mass. ${ }^{4}$ In rat pancreatic $\beta$-cells, imeglimin decreased basal apoptosis in the absence of cytokines by $10 \% .^{7}$ In the presence of cytokines, imeglimin has been seen to diminish the pro-apoptotic effect due to the cytokines, and demonstrated a reduction in apoptotic cell death by $37 \%$ and $25 \%$, at $0.1 \mathrm{mmol} / \mathrm{L}$ and $1 \mathrm{mmol} / \mathrm{L}$, respectively. ${ }^{?}$ In addition, imeglimin has shown a large protective effect in glucose-induced toxicity. ${ }^{4}$ The molecular pathways behind these actions are mainly related to the mitochondria, and the proposed mechanism is the change in permeability transition pores of mitochondria. Imeglimin prevents hyperglycaemia-induced cell death in human microvascular endothelial cells-1 (HMEC-1) through the inhibition of permeability transition pores opening without inhibiting mitochondrial respiration or affecting cellular energy status., ${ }^{3,4}$

\section{Additional effects and benefits}

The protection from ROS as a result of imeglimin's action may also be extended to other organs. A study indicated that imeglimin may present cardiovascular benefit, as it has shown promising signs in rats with metabolic syndrome. In this study, improvement in cardiac function and increased left ventricle tissue perfusion were reported. These improvements were associated with a reduction in ROS production by the left ventricle, an increase in coronary artery endothelium-dependent relaxation and a reduction in albuminuria, which was followed by a decrease in renal interstitial fibrosis. Rates of tubular injury and interstitial inflammation, however, did not seem to change significantly. ${ }^{9}$

\section{Clinical studies}

Metformin is the first-line agent in the treatment of type 2 diabetes, mainly due to its safety, efficacy and low cost. It does not cause hypoglycaemia, and offers an effective reduction in HbA1C as a monotherapy or in combination with other oral glucose-lowering agents. However, in some patients, the use of metformin is limited, mostly due to its gastrointestinal side effects. ${ }^{10}$ There are several phase II and phase III studies that demonstrate the effectiveness of imeglimin as an alternative option.

In a 4-week phase Ila, three-arm parallel group trial in 59 patients with type 2 diabetes by Pirags et al., imeglimin was compared with metformin and placebo based on safety profile and efficacy to reduce the plasma glucose concentration $\mathrm{A} \cup \mathrm{C}\left(\mathrm{A} \cup \mathrm{C}_{\mathrm{PG}}\right)$. The patients $(\mathrm{HbA} 1 \mathrm{C}$ : 6.5-8.5\%) either did not receive any treatment at all, or were treated with monotherapy sulfonylurea or metformin before the initiation of the study, and were randomized to imeglimin 2,000 mg once daily, imeglimin 1,000 mg twice daily (BID), metformin $850 \mathrm{mg}$ BID (mean baseline HbA1C levels of $7.41,7.07$ and $7.27 \%$, respectively), or placebo. Imeglimin BID presented the greatest reduction in $\mathrm{AUC}_{\mathrm{PG}}$ from baseline, followed by metformin and imeglimin once daily dosing $(-33 \%,-30 \%$ and $-10 \%$, respectively). The results of this trial indicated that imeglimin presented a similar efficacy to metformin. ${ }^{11}$

In a second 8-week phase Ila, four-arm, controlled multicentre study by Pirags et al. in 128 patients with type 2 diabetes, patients who were treatment naïve or received a sulfonylurea or metformin as a monotherapy were randomized to imeglimin 500 mg BID, imeglimin 1,500 mg BID, metformin $850 \mathrm{mg}$ BID or placebo (mean baseline HbA1C levels of $7.20 \%, 7.35 \%, 7.12 \%$ and $7.21 \%$, respectively). Imeglimin $1,500 \mathrm{mg} \mathrm{BID}$ and metformin BID were superior to placebo in the assessment of the AUC up to 6 hours $\left(\mathrm{AUC}_{0-6 \mathrm{~h}}\right.$ ) for glucose during a prolonged meal, and both led to the reduction of FPG and $\mathrm{HbA} 1 \mathrm{C}$ from baseline; imeglimin 500 mg BID was the least effective dosing, and led to an increase of FPG and $\mathrm{HBA} 1 \mathrm{C}$ from the baseline across all imeglimin groups. Concerning the safety profile of imeglimin, only 16 patients experienced headache compared with 20 patients in the metformin group, and gastrointestinal side effects were mainly observed in the metformin group. ${ }^{11}$ Although these studies highlight the efficacy of imeglimin based on specific glycaemic parameters, they cannot reliably examine the true effect of imeglimin on $\mathrm{HbA} 1 \mathrm{C}$, which represents a 3-month blood glucose average.2 
In a multicentre, randomized controlled trial, 156 patients (HDA1C >7.5\%) were randomized $1: 1$ to receive imeglimin $1,500 \mathrm{mg}$ BID or placebo, added to a stable dose of metformin (1,500-2,000 mg/day). At 12 weeks, patients on combination metformin + imeglimin therapy had a significant decrease from baseline in both $\mathrm{HbA} 1 \mathrm{C}$ (8.5-7.8\% versus 8.6-8.3\%) and FPG (10.4-9.5\% versus 10.3-10.6\%) when compared with the metformin + placebo group. Metformin + imeglimin therapy was well tolerated, with a similar safety profile to metformin + placebo..$^{12}$ In a second multicentre, randomized, placebo-controlled study, conducted by Fouqueray et al., 170 patients ( $\mathrm{HbA} 1 \mathrm{C}>7.5 \%)$ on sitagliptin monotherapy were randomized to receive sitagliptin + placebo or sitagliptin + imeglimin 1,500 mg BID for 12 weeks. In the imeglimin arm, there was a notable decrease in $\mathrm{HbA} 1 \mathrm{C}(-0.6 \%)$ from baseline (8.5\%), compared with an increase of $0.12 \%$ in the placebo group (baseline HbA1c: $8.47 \%$ ). There was also a significant decrease in FPG $(-0.93 \%$ from baseline $(10.53 \%)$, compared with a decrease of $0.11 \%$ in the placebo group. The reported side effects in the imeglimin group were similar to those in the placebo group. ${ }^{13}$ There were also no significant differences in the mean changes from baseline to week 12 between treatment groups for triglyceride and C-reactive protein levels, as well as systolic blood pressure. Based on these studies, it may be concluded that imeglimin BID dosing is more effective than once-daily dosing, and can be used efficiently in combination with metformin or sitagliptin without any major side effects compared with placebo..$^{12,13}$

In a recent larger study that included 299 Japanese adults with diabetes who were treatment naive or previously treated with only one oral antidiabetic medication, the efficacy and safety profile of imeglimin as a monotherapy was assessed compared with placebo. ${ }^{14}$ In this 24-week, randomized, double-blind, parallel group, dose-ranging, phase IIb clinical trial, the participants were randomized (1:1:1:1) to the following: imeglimin 500 mg, imeglimin 1,000 mg, imeglimin 1,500 mg or placebo BID. At week 24, imeglimin considerably reduced $\mathrm{HbA} 1 \mathrm{c}$ versus placebo (imeglimin 500 mg versus placebo: -0.52\%; imeglimin 1,000 mg versus placebo: $-0.94 \%$; imeglimin 1,500 mg versus placebo: $-1.00 \%)$. Adverse events were observed in $68.0 \%, 62.2 \%, 73.3 \%$ and $68.0 \%$ of the patients receiving imeglimin $500 \mathrm{mg}$, imeglimin 1,000 mg, imeglimin 1,500 mg and placebo, respectively. The most common adverse events were infections and infestations, with a slight increase over placebo in all imeglimin groups. The second most common adverse events were gastrointestinal disorders. The proportion of patients reporting gastrointestinal events was higher in the imeglimin 1,500 mg group compared with the placebo and other imeglimin dosage groups; there was a higher proportion of diarrhoea, abdominal discomfort, nausea and vomiting at the dose of $1,500 \mathrm{mg}$. Hypoglycaemic events were balanced between the groups. According to this trial, imeglimin was proven to be well tolerated as a monotherapy, significantly boosting glycaemic control by reducing $\mathrm{HbA} 1 \mathrm{C}$, compared with the placebo. ${ }^{14}$

The approval of imeglimin in Japan is supported by a phase III clinical programme, which includes three trials: TIMES 1, TIMES 2 and TIMES 3 (Trials of imeglimin for efficacy and safety). ${ }^{15-17}$ In TIMES 1, which was a randomized, double-blind, placebo-controlled monotherapy study that included 213 Japanese patients (mean age 62 years) with type 2 diabetes, orally administered imeglimin (1,000 mg BID) was compared with placebo for 24 weeks. In the imeglimin group, there was a notably greater reduction in HbA1c compared with the placebo group (placebo group mean $\mathrm{HbA} 1 \mathrm{C}$ at baseline $7.93 \%$ ), with an HbA1c placebo-corrected mean change from the baseline of $-0.87 \%$. The decrease in FPG level was also higher among patients who received imeglimin versus placebo (placebo-adjusted least squares [LS] mean decrease of $19 \mathrm{mg} / \mathrm{dL}$ in the imeglimin group). In TIMES 1, only $44.3 \%$ of the patients treated with imeglimin and $44.9 \%$ of those given placebo reported adverse effects, while $2.8 \%$ and $5.6 \%$, respectively, experienced side effects resulting in treatment discontinuation. ${ }^{15}$

The TIMES 2 and TIMES 3 trial results were recently presented at the 56th European Association for the Study of Diabetes annual meeting. ${ }^{16,17}$ In TIMES 2, a 52-week, open-label, parallel-group trial, imeglimin $1,000 \mathrm{mg}$ BID was orally administered to 714 Japanese patients with type 2 diabetes as monotherapy or as an add-on to stable doses of oral or approved, injectable hypoglycaemic therapies. The respective changes in mean $\mathrm{HbA} 1 \mathrm{C}$ values from baseline at 52 weeks after co-administration of imeglimin and the other studied hypoglycaemic agents were as follows: dipeptidyl peptidase-4 inhibitors $(-0.92 \%)$, thiazolidinediones $(-0.88 \%)$, alpha-glucosidase inhibitors $(-0.85 \%)$, glinides $(-0.70 \%)$, metformin $(-0.67 \%)$, sodium-glucose transport protein 2 inhibitors $(-0.57 \%)$, sulphonylureas (-0.56\%), glucagon-like peptide 1 receptor agonists $(-0.12 \%)$, imeglimin monotherapy $(-0.46 \%){ }^{16}$

In the TIMES 3 trial, the combination of imeglimin and insulin was studied in a 16-week, double-blind, placebo-controlled, randomized trial of 215 Japanese patients with type 2 diabetes who were insufficiently controlled on insulin therapy (mean $\mathrm{HbA} 1 \mathrm{c}$ placebo-corrected reduction from baseline at 16 weeks: $-0.60 \%, p<0.0001$; primary endpoint). Consequently, in a 36-week, open-label extension period that was not placebo-controlled, 208 participants who completed the first 16-week, placebo-controlled phase of the study received imeglimin 1,000 mg BID orally and insulin. In the group receiving imeglimin and insulin for 16 weeks and imeglimin and insulin for the next 36 weeks, a decrease of $0.64 \%$ mean $\mathrm{HbA} 1 \mathrm{C}$ versus the baseline was observed. Lastly, in the group that received placebo and insulin for the first 16 weeks, followed by co-administration of imeglimin and insulin on the extension period, there was a change of $-0.54 \%$, compared with the baseline. ${ }^{17}$

In both TIMES 2 and TIMES 3, the adverse events were similar to previous clinical trials; overall, the safety profile of imeglimin was favourable and the adverse event incidence was similar to that of a placebo. Hypoglycaemic events reported during TIMES 3 were mild, and the number of patients receiving imeglimin who experienced hypoglycaemia was similar to the placebo group. ${ }^{16,17}$

\section{Discussion}

The mechanisms by which imeglimin improves insulin sensitivity and $\beta$-cell function are not yet fully understood. The examination of imeglimin's effect on inflammatory responses, glucagon secretion and mitochondrial function may reveal other pharmacological aspects that demonstrate the potential of this promising new molecule. So far, only a few large, phase III clinical trials have been conducted to evaluate the efficacy of imeglimin as monotherapy or in combination with other approved antidiabetic agents. So far, imeglimin has exhibited a good safety profile in most of the recent clinical trials; it has shown a lower frequency of gastrointestinal adverse effects than metformin, while demonstrating a similar efficacy on glycaemic control, both as a monotherapy and in combination with other antidiabetic medications. ${ }^{2}$ In patients with moderate hepatic impairment, imeglimin administration was well tolerated. ${ }^{6}$ Although the increase on maximum plasma concentration in these individuals was not reported as clinically significant, more research needs to be conducted in order to examine the safety profile on individuals with severe hepatic impairment.

This review presents a general overview of imeglimin, including its mechanism of action, and the most important studies in the clinical 
development programme of this promising agent. In contrast with a However, the appraisal of the most relevant research about this topic systematic review, this paper aims to introduce this under-development summarizes the current lack of knowledge and demonstrates the need agent to the medical community in a comprehensive, narrative way. for future research.

1. Vuylsteke V, Chastain LM, Maggu GA, Brown C. Imeglimin: A potential $V$, Ch multin LM, M drug for type 2 diabetes. Drugs R D. 2015;15:227-32.

2. Yendapally R, Sikazwe D, Kim SS, et al. A review of phenformin, metformin, and imeglimin. Drug Dev Res. 2020;81:390-401.

3. Yaribeygi $\mathrm{H}$, Maleki M, Sathyapalan T, et al. Molecular mechanisms by which imeglimin improves glucose homeostasis. J Diabetes Res. 2020;2020:8768954.

4. Hallakou-Bozec S, Vial G, Kergoat M, et al. Mechanism of action of Imeglimin: A novel therapeutic agent for type 2 diabetes. Diabetes Obes Metab. 2021;23:664-73.

5. Clémence C, Fouqueray P, Sébastien B. In vitro investigation, pharmacokinetics, and disposition of imeglimin, a novel oral antidiabetic drug, in preclinical species and humans. Drug Metab Dispos. 2020;48:1330-46.

6. Chevalier C, Dubourg J, Bolze S, Fouqueray P. Pharmacokinetics of imeglimin in subjects with moderate hepatic impairment. Clin Pharmacokinet. 2021;60:485-90.

7. Fouqueray $P$, Leverve $X$, Fontaine $E$, et al. Imeglimin - a new Fouqueray $P$, Leverve $X$, Fontaine $E$, et al. Imeglimin - a new
oral anti-diabetic that targets the three key defects of type 2 diabetes. J Diabetes Metab. 2011;2:4.

8. Perry RJ, Cardone RL, Petersen MC, et al. Imeglimin lowers glucose primarily by amplifying glucose-stimulated insulin secretion in high-fat-fed rodents. Am J Physiol Endocrino Metab. 2016:311:E461-70

9. Lachaux M, Soulié M, Hamzaoui M, et al. Short-and long-term administration of imeglimin counters cardiorenal dysfunction in a rat model of metabolic syndrome. Endocrinol Diabetes in a rat model of metab.

10. American Diabetes Association. 9. Pharmacologic approaches to glycemic treatment: standards of medical care in diabetes-2020. Diabetes Care. 2020;43(Suppl. 1):S98-110

11. Pirags V, Lebovitz H, Fouqueray P. Imeglimin, a novel glimin oral antidiabetic, exhibits a good efficacy and safety profile in type 2 diabetic patients. Diabetes Obes Metab. 2012;14:852-8.

12. Fouqueray $P$, Pirags $V$, Inzucchi $S E$, et al. The efficacy and safety of imeglimin as add-on therapy in patients with type 2 diabetes inadequately controlled with metformin monotherapy. Diabetes Care. 2013;36:565-8.

13. Fouqueray $\mathrm{P}$, Pirags $\mathrm{V}$, Diamant $\mathrm{M}$, et al. The efficacy and safety of imeglimin as add-on therapy in patients with type 2 diabetes inadequately controlled with sitagliptin monotherapy. Diabetes Care. 2014:37:1924-30

14. Dubourg J, Ueki K, Grouin J-M, Fouqueray P. Efficacy and safety of imeglimin in Japanese patients with type 2 diabetes mellitus:
A 24-week, randomized, double-blind, placebo-controlled, dose-ranging phase $2 \mathrm{~b}$ trial.

15. Poxel. Poxel announces detailed imeglimin phase 3 TIMES 1 results presented at 55th Annual Meeting of the European Association for the Study of Diabetes. 2019. Available at: www.poxelpharma.com/en_us/investors/news-events/ press-releases/detail/128/poxel-announces-detailed-imegliminphase-3-times-1-results (accessed 19 May 2021).

16. Poxel. Poxel presents imeglimin phase 3 TIMES results and PXL770 preclinical cardio-renal results at the 56th European Association for the Study of Diabetes Annual Meeting. 2020. Available at: www.poxelpharma.com/en_us/investors/ news-events/press-releases/detail/163/poxel-presentsimeglimin-phase-3-times-results-and-px|770 (accessed 19 May 2021).

17. Poxel Pharma. Poxel announces positive topline results for imeglimin phase 3 trial (TIMES 3 36-week) for the treatment of type 2 diabetes in Japan. [Press release]. 2019. Available at: www.poxelpharma.com/en_us/news-media/press-releases/ www.poxelpharma.com/en_us/news-media/press-releases/
detail/136/poxel-announces-positive-topline-results-fordetail/136/poxel-announces-positive-t
imeglimin (accessed 24 October 2021) 